\title{
Metabolic marker gene mining provides insight in global mcrA diversity and, coupled with targeted genome reconstruction, sheds further light on metabolic potential of the Methanomassiliicoccales
}

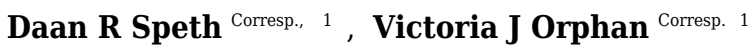 \\ ${ }^{1}$ Geological and Planetary Sciences, California Institute of Technology, Pasadena, CA, United States \\ Corresponding Authors: Daan R Speth, Victoria J Orphan \\ Email address: dspeth@caltech.edu, vorphan@gps.caltech.edu
}

Over the past years, metagenomics has revolutionized our view of microbial diversity. Moreover, extracting near-complete genomes from metagenomes has led to the discovery of known metabolic traits in unsuspected lineages. Genome-resolved metagenomics relies on assembly of the sequencing reads and subsequent binning of assembled contigs, which might be hampered by strain heterogeneity or low abundance of a target organism. Here we present a complementary approach, metagenome marker gene mining, and use it to assess the global diversity of archaeal methane metabolism through the $m c r A$ gene. To this end, we have screened 18,465 metagenomes for the presence of reads matching a database representative of all known mcrA proteins and reconstructed gene sequences from the matching reads. We use our mcrA dataset to assess the environmental distribution of the Methanomassiliicoccales and reconstruct and analyze a draft genome belonging to the 'Lake Pavin cluster', an uncultivated environmental clade of the Methanomassiliicoccales. Analysis of the 'Lake Pavin cluster' draft genome suggests that this organism has a more restricted capacity for hydrogenotrophic methylotrophic methanogenesis than previously studied Methanomassiliicoccales, with only genes for growth on methanol present. However, the presence of the soluble subunits of methyltetrahydromethanopterin:CoM methyltransferase ( $m t r A H)$ provide hypothetical pathways for methanol fermentation, and aceticlastic methanogenesis that await experimental verification. Thus, we show that marker gene mining can enhance the discovery power of metagenomics, by identifying novel lineages and aiding selection of targets for in-depth analyses. Marker gene mining is less sensitive to strain heterogeneity and has a lower abundance threshold than genome-resolved metagenomics, as it only requires short contigs and there is no binning step. Additionally, it is computationally cheaper than genome resolved metagenomics, since only a small subset of reads needs to be assembled. It is therefore a suitable approach to extract knowledge from the many 
publicly available sequencing projects. 
1 Metabolic marker gene mining provides insight in global mcrA diversity and, coupled with targeted

2 genome reconstruction, sheds further light on metabolic potential of the Methanomassiliicoccales

3

4 Daan R. Speth ${ }^{1}$ and Victoria J. Orphan ${ }^{1}$

5 'Division of Geological and Planetary Sciences, California Institute of Technology, Pasadena, CA, USA

6

7 Correspondence:

8 Daan R. Speth

9 dspeth@caltech.edu

10 or

11 Victoria J. Orphan

12 vorphan@gps.caltech.edu

13

14 


\section{Abstract}

16 Over the past years, metagenomics has revolutionized our view of microbial diversity. Moreover,

17 extracting near-complete genomes from metagenomes has led to the discovery of known metabolic traits

18 in unsuspected lineages. Genome-resolved metagenomics relies on assembly of the sequencing reads and

19 subsequent binning of assembled contigs, which might be hampered by strain heterogeneity or low

20 abundance of a target organism. Here we present a complementary approach, metagenome marker gene

21 mining, and use it to assess the global diversity of archaeal methane metabolism through the mcrA gene.

22 To this end, we have screened 18,465 metagenomes for the presence of reads matching a database

23 representative of all known mcrA proteins and reconstructed gene sequences from the matching reads.

24 We use our mcrA dataset to assess the environmental distribution of the Methanomassiliicoccales and

25 reconstruct and analyze a draft genome belonging to the 'Lake Pavin cluster', an uncultivated

26 environmental clade of the Methanomassiliicoccales. Analysis of the 'Lake Pavin cluster' draft genome

27 suggests that this organism has a more restricted capacity for hydrogenotrophic methylotrophic

28 methanogenesis than previously studied Methanomassiliicoccales, with only genes for growth on

29 methanol present. However, the presence of the soluble subunits of

30 methyltetrahydromethanopterin:CoM methyltransferase ( $m t r A H)$ provide hypothetical pathways for

31 methanol fermentation, and aceticlastic methanogenesis that await experimental verification. Thus, we

32 show that marker gene mining can enhance the discovery power of metagenomics, by identifying novel

33 lineages and aiding selection of targets for in-depth analyses. Marker gene mining is less sensitive to strain

34 heterogeneity and has a lower abundance threshold than genome-resolved metagenomics, as it only

35 requires short contigs and there is no binning step. Additionally, it is computationally cheaper than

36 genome resolved metagenomics, since only a small subset of reads needs to be assembled. It is therefore

37 a suitable approach to extract knowledge from the many publicly available sequencing projects. 


\section{Introduction}

40 Genome resolved metagenomics is allowing unprecedented, primer independent, insight in the diversity

41 of the microbial world (Tyson et al., 2004; Hug et al., 2016). In addition to the window into microbial

42 diversity that metagenomics sequencing offers, it also provides clues for the metabolism of the organisms

43 observed (Tyson et al., 2004). More precisely, based on the presence (or absence) of homologs of

44 previously studied genes, an educated guess of the metabolism of an organism can be made. This has

45 amongst others, led to the recent discovery of complete ammonium oxidation (comammox) in a single

46 organism (van Kessel et al., 2015; Daims et al., 2015) and provided evidence for archaeal methane

47 metabolism outside of the Euryarchaeota (Evans et al., 2015; Vanwonterghem et al., 2016).

48 Other major advances in our understanding of the diversity of archaeal methane metabolism have come

49 from cultivation studies, including the culturing and enrichment of members of the 7th Euryarchaeal order

50 of methanogens, the Methanomassiliicoccales (Dridi et al., 2012; Borrel et al., 2012; lino et al., 2013;

51 Borrel et al., 2013a), and the recent culturing of halophilic methanogens from Siberian soda lakes (Sorokin

52 et al., 2017). The latter group seems to be restricted to highly saline environments, whereas

53 environmental sequencing indicates that the Methanomassiliicoccales are widely distributed, occurring in

54 habitats ranging from animal guts to wetlands and wastewater treatment (Großkopf et al., 1998; Tajima

55 et al., 2001; Wright et al., 2004; lino et al., 2013; Söllinger et al., 2016). Indeed, a recent large-scale effort

56 to bin genomes from environmental metagenome data recovered 66 Methanomassiliicoccales genomes

57 (Parks et al., 2017), further supporting the environmental relevance of this order. It has recently been

proposed that the Methanomassiliicoccales should be divided into an environmental clade and a gastrointestinal tract (GIT) clade (Söllinger et al., 2016). In addition, a 'Lake Pavin' clade, named after the

60 site where it was first detected, was previously proposed based on analysis of environmental $16 \mathrm{~S}$

61 ribosomal RNA gene sequencing (Borrel et al., 2013b). Another recent study suggested the existence of

62 Methanomassiliicoccales in marine sediments, based on the presence of butanetriol dibiphytanyl glycerol 
63 tetraether (BDGT) lipids in Methanomassiliicoccus luminyensis, and the detection of these lipids in marine

64 sediments (Becker et al., 2016), but the specificity of this biomarker is unclear.

65 The recent advances in our understanding of the diversity of archaeal methane metabolism raise the

66 question whether additional novel diversity exists within previously sequenced metagenomic datasets,

67 and whether the environmental importance and diversity of understudied clades can be further

68 illuminated. In the examples of metagenomics enabled discovery discussed above, homologs of marker

69 genes known to be diagnostic for methane metabolism were discovered in metagenome assembled

70 genomes (MAGs). This requires the assembly of the raw sequencing reads, and subsequent binning of the

71 assembled contigs into draft genomes (Dick et al. 2009; Thomas et al., 2012). Assembly is computationally

72 expensive on large datasets, and strain diversity within a sample can result in highly fragmented

73 assemblies (Thomas et al., 2012). Automated binning has improved dramatically in recent years, but this

74 process often still requires substantial time-consuming manual curation (Albertsen et al, 2013; Delmont

75 et al., 2017). Alternatively, the diversity of organisms capable of a metabolic process can be assessed using

76 PCR-based screening of environmental samples. However, PCR-based analyses are sensitive to primer

77 bias, and therefore unlikely to yield highly divergent gene sequences. In addition, the amplification of a

78 single metabolic gene makes elucidation of the taxonomic affiliation of the organism containing the gene

79 difficult, although for some well-studied marker genes the coupling between gene and organism

80 phylogeny has been documented.

81 Directly mining metagenomic reads for marker genes, and subsequently reconstructing the full-length

82 gene sequence, combines some of the advantages of both of these strategies, while minimizing the

83 disadvantages. Using a curated database, reads can be confidently assigned to a gene of interest, with

84 false positive removal using a BLAST Score Ratio (BSR) (Rasko et al., 2005). If a divergent variant of a gene

85 of interest is retrieved using this approach, the genome containing this gene can be retrieved from the

86 source metagenome dataset using targeted (manual) binning. This last step will not always be successful, 
87 because less sequencing depth is required to assemble a single short contig consisting of one gene, rather

88 than assemble longer contigs and confidently assign them to a draft genome. However, precisely because

89 more sequencing depth is required to assemble and bin a draft genome than a single gene, marker gene

90 mining might yield information from datasets where genome binning is not feasible (e.g. Lüke et al., 2016).

91 We have previously used this approach to assess the presence of nitrogen cycle genes in datasets from

92 the Arabian Sea oxygen minimum zone (Lüke et al., 2016) and to assess the environmental distribution of

93 organisms capable of complete ammonium oxidation (van Kessel et al., 2015). Here we present a more

94 systematic use of marker gene mining to assess the diversity of the $m c r A$ gene, encoding the alpha subunit

95 of the methyl-coenzyme $M$ reductase. This enzyme is essential for (reverse) methanogenesis, where it

96 catalyzes the final reduction and release of the methyl group on coenzyme $M$ to methane, or the initial

97 oxidation of methane (Nagle and Wolfe, 1983; Scheller et al., 2010). Moreover, the mcrA gene has recently

98 been discovered in several unexpected clades of Archaea, indicating that methane metabolism is more

99 widespread in the domain than previously thought (Mondav et al., 2014; Evans et al., 2015;

100 Vanwonterghem et al., 2016; Sorokin et al., 2017). Notably, Syntrophoarchaeum, an archaeon in a

101 syntrophic anaerobic butane-oxidizing enrichment culture, contained multiple copies of highly divergent

102 mcrA genes thought to be involved in the activation of higher alkanes, but not methane (Laso-Pérez et al.,

103 2016).

104 We have screened the environmental metagenomic data available in the NCBI sequencing read archive

105 (Kodama et al., 2012) and MG-RAST (Meyer et al., 2008) for reads matching the mcrA gene. We

106 subsequently used the obtained data to assess the diversity and environmental distribution of the

107 Methanomassiliicoccales order (Paul et al., 2012). Finally, we reconstruct and analyze a draft genome of

108 an organism belonging to the 'Lake Pavin cluster' an uncultivated lineage within this group (Borrel et al.,

109 2013b).

110 


\section{Methods}

112

113 Construction of the mcrA database from PFam and NCBI-nr

114 To construct a mcrA protein sequence database representative of the known global diversity, we first

115 obtained the amino acid sequences in the Pfam (version 29.0; Finn et al., 2013) families MCR_alpha_N

116 (PF02745) and MCR_alpha (PF02249), representing the N-terminal and C-terminal parts of the mcrA

117 protein. We assessed the completeness of the Pfam dataset by downloading the NCBI-nr protein

118 reference database in fasta format (ftp://ftp.ncbi.nlm.nih.gov/blast/db/FASTA/) and using it as query for

119 a DIAMOND (Buchfink et al., 2014) search against the Pfam dataset. Subsequently, we calculated the

120 BLAST score ratio (BSR) (Rasko et al., 2005) between the score against the Pfam dataset and the maximum

121 possible score (a self-hit) of the 16,260 sequences that had a DIAMOND hit against the Pfam dataset

122 (Supplemental Figure 1). This allowed us to identify real mcrA sequences not covered in the Pfam, while

123 eliminating false positive hits. Using this method, we identified that mcrA sequences from the clades

124 Bathyarchaeota, Methanofastidiosales (WSA2), ANME-1, Methermicoccus and Methanoperedens (ANME-

125 2d) were not represented in the Pfam at the time of database construction (June 2016) (Supplemental

126 Figure 1). All 203 full-length mcrA sequences in the NCBI-nr were added to the mcrA dataset, which was

127 subsequently clustered at $90 \%$ identity using UCLUST (Edgar, 2010), resulting in a mcrA reference

128 database containing 69 non-redundant full-length sequences representing the full diversity of mcrA

129 sequences included in the NCBI-nr (June 2016).

130

131 mcrA read data acquisition from SRA and MG-RAST

132 To obtain a list of metagenome datasets of potential interest, metadata was downloaded for all runs in

133 the sequencing read archive (SRA) (https://www.ncbi.nlm.nih.gov/sra) corresponding to the query

134 "metagenomic AND WGS NOT human NOT gut NOT oral" (gut and oral were excluded because of the high 
135 number of datasets from these environments) on June 16th 2016. Additionally, metadata for all runs in

136 MG-RAST (https://metagenomics.anl.gov/) was downloaded, and all datasets labeled 'WGS' (whole

137 genome shotgun) were selected. This resulted in a list of 10,613 SRA run accession numbers and 7,852

138 MG-RAST identifiers, representing over 60 Terabases of sequencing data (Figure 1, Supplemental files 1

139 and 2). As storing this amount of sequence data was not feasible, the accession lists were used as input

140 for the 'sra_trawler.sh' and 'mgrast_trawler.sh' shell scripts, respectively. Briefly, these scripts download

141 an accession number on the list, then use the downloaded dataset as query in a DIAMOND (Buchfink et

142 al., 2014) search against a database of interest (in this case the mcrA protein database described above).

143 Then, hits are written to a new file in fasta format using the script 'blast_based_read_lookup.pl', the

144 dataset is discarded, and the process is repeated for the next dataset. Scripts are available at

145 (https://github.com/dspeth/bioinfo_scripts/tree/master/metagenome_screening). To speed up the

146 process, the sra-trawler.sh script uses the sra-toolkit

147 (https://www.ncbi.nlm.nih.gov/sra/docs/toolkitsoft/) to split paired-end files and only analyzes the

148 forward reads of paired-end datasets. Using this approach, it took approximately 4 months to process the

14918,465 selected datasets using 16 cores on a server. 2,083,349 reads from 6,105 datasets matched the

150 mcrA database and were combined and used as the query in a DIAMOND search against the NCBI-nr

151 database to calculate the BSR between the hit against the mcrA database and the NCBI-nr (Figure 1). This

152 results in a BSR between the score against the database of interest and the score against an outgroup,

153 rather than a BSR between the score against the database of interest and a self-hit described above for

154 mcrA database construction. Applying BSR in this manner allows for detection of real mcrA sequences

155 with low identity to the database while still removing false positives (Lüke et al., 2016). The resulting

$1562,081,632$ post-BSR hits originated from 3,684 datasets (Figure 1). Dataset SRR398144, an mcrA amplicon

157 sequencing effort (Denonfoux et al., 2013), accounted for nearly $10 \%$ of these hits $(197,371)$. As the aim 
158 of our work was to reconstruct near full-length mcrA sequences, SRR398144 was excluded and the

159 remaining steps were done with 1,884,261 reads.

160

161 Assessing global mcrA protein diversity

162 The true-positive reads from 1,080 datasets containing over 20 hits, enough for approximately 2-fold gene

163 coverage in a 150bp dataset, were assembled separately using IDBA-UD (Peng et al., 2012), resulting in

1641,511 sequences $>1,000$ bp. Prodigal (Hyatt et al., 2010; v2.6.2) was used in single mode and without the

$165-\mathrm{c}$ flag, allowing open ends, for open reading frame prediction on the assembled contigs and mcrA protein

166 sequences over 300 amino acids were selected. The resulting 1,460 sequences were sorted by length and

167 added to the mcrA database. Additionally, the eight recently published Syntrophoarchaeum mcrA

168 sequences (Laso-Pérez et al., 2016, not available at the time of initial database construction and mining)

169 were added to the mcrA database, and the new set was clustered using UCLUST at 90\% identity (Edgar,

170 2010). The resulting 150 sequences were aligned using MUSCLE (Edgar, 2004) and a maximum likelihood

171 phylogeny was calculated using RAxML (Stamatakis, 2014), with the LG4X substitution model (Le et al.

172 2012) and 500 bootstrap replicates. The phylogeny was visualized using iTOL (Letunic and Bork, 2016;

173 Figure 2). To estimate previous detection of the mcrA sequences, all 15,888 hits post-BSR selection in the

174 NCBI-nr that were too short to be included in the database (PCR products) were used as DIAMOND query

175 against the 150 non-redundant mcrA sequences (Supplemental Figure 2A). Additionally, all 1,884,261

176 metagenome hits were used as DIAMOND query against the 149 non-redundant mcrA sequences

177 (Supplemental Figure 2B). Relative counts of all 13,007 NCBI-nr hits and 1,487,226 metagenomic reads

178 over $90 \%$ identity (as these would be clustered with the sequence) were visualized using iTOL.

179 Reconstructed gene sequences are included as supplemental file 3 and 4, containing the nucleotide and 180 amino acid sequences respectively. 


\section{Environmental distribution of the Methanomassiliicoccales}

183 The assembled mcrA sequences belonging to the Methanomassiliicoccales clade were obtained using BSR

184 between the best hit against the five Methanomassiliicoccales mcrA sequences present in our mcrA

185 database, before addition of the newly assembled sequences (Figure 2), and the best hit against our entire

186 mcrA database. Sequences with a BSR over 0.75 were assigned to the Methanomassiliicoccales

187 (Supplemental Figure 2). The resulting 116 sequences, combined with the five reference sequences

188 discussed above and a Methanofastidiosales sequence (KYC45731.1, as outgroup), were aligned using

189 MUSCLE (Edgar, 2004) and a phylogeny was calculated using RaxML (Stamatakis, 2014) with the LG4X

190 substitution model (Le et al. 2012) and 500 bootstrap replicates. The resulting phylogeny was visualized

191 using iTOL (Letunic and Bork, 2016). The source environment of each sequence was assigned manually,

192 using the NCBI-SRA or MG-RAST sample record.

193

194 Genome reconstruction of a Lake Pavin cluster Methanomassiliicoccales

195 Reads from dataset SRR636597 (Tan et al., 2015) were assembled using Megahit (Li et al., 2015), (v.1.0.3,

196 with --presets meta-large) resulting in 125,408 contigs longer than 1,000bp. Reads from datasets

197 SRR636597, SRR636559, and SRR636569, which contained highly similar mcrA sequences (Figure 3), were

198 mapped to the assembled contigs using BBmap (http://jgi.doe.gov/data-and-tools/bbtools/bb-tools-user-

199 guide/) using a cutoff of $95 \%$ identity over $80 \%$ of the read length. Kmer frequency of the contigs was

200 calculated using the script calc.kmerfreq.pl by Mads Albertsen (https://github.com/MadsAlbertsen/multi-

201 metagenome/tree/master/R.data.generation). The contig fasta file was converted to tab-delimited form

202 and length and GC content were calculated using fasta_to_gc_length_tab.pl

203 (https://github.com/dspeth/bioinfo_scripts/tree/master/metagenome_screening). The contig containing

204 the mcrA sequence of interest was identified using DIAMOND (Buchfink et al., 2014) and used to guide

205 the manual binning of the cluster representing the draft genome containing this gene, using $\mathrm{R}$, as 
206 previously described (Speth et al., 2016). The resulting $1.6 \mathrm{Mbp}$ draft genome was further refined by 10

207 cycles of mapping with BBmap and assembly with SPAdes (Bankevich et al., 2012) using the

208 iterative_denovo_spades.sh shell script

209 (https://github.com/dspeth/bioinfo_scripts/tree/master/metagenome_screening). A final manual

210 refining was performed using anvi'o v4, using clustering based on composition of the contigs as well as

211 their coverage in datasets SRR636597, SRR636559, and SRR636569 to remove contaminating contigs from

212 the bin (Eren et al. 2015). The final 1.55Mb draft genome on 58 contigs was quality checked using checkM

213 (v1.0.6) with the default settings of the lineage_wf command (Parks et al., 2015), annotated using Prokka

214 (v1.12), with flags --compliant, --metagenome, and --kingdom Archaea (Seemann, 2014), analyzed using

215 Artemis (Carver et al., 2012), compared to the other available Methanomassiliicoccales genomes using

216 ProteinOrtho with default settings (Lechner et al., 2011; v5.12), and the annotation was manually curated

217 by verifying the presence/absence of the complexes discussed in the text. As NCBI does not accept third

218 party annotation without experimental validation (see: https://www.ncbi.nlm.nih.gov/genbank/tpa/),

219 the assembled annotated draft genome is included as supplemental file 5. 


\section{Results and discussion}

\section{McrA gene diversity across all sampled datasets}

224 To leverage the available metagenomic sequencing data for a diversity analysis of functional marker 225 genes, we established a workflow based on automated sequential downloading and processing of the 226 public data in the Sequencing Read Archive (SRA) and the Metagenomics RAST (MG-RAST) repositories

227 (Figure 1). We applied this workflow to the mcrA gene, a marker for the production and anaerobic 228 oxidation of methane, because of the environmental relevance of these processes (Knittel and Boetius, 229 2009). The recently discovered mcrA sequences of the Verstraetearchaeota, Syntrophoarchaea, 230 Methanonatronarchaeia, and Methanoflorens were not present in the NCBI-nr as of June 2016, and thus 231 not included in our reference database. Of these, our analysis does retrieve mcrA sequences associated 232 with Verstraetearcheota, Methanonatronarchaeia, and Methanoflorens (Figure 2), but was not sensitive 233 enough to retrieve sequences related to the highly divergent Syntrophoarchaea, simultaneously

234 illustrating both the power to detect novel diversity, and the limit of screening of metagenomic reads 235 based on sequence identity. The HMM based search strategy implemented in GraftM (Boyd et al., 2018), 236 offers a complementary strategy for mining unassembled reads, further increasing the potential for 237 discovery of novel diversity.

238 Besides the independent recovery of the Verstraetearcheota, Methanonatronarchaeia, and 239 Methanoflorens from public databases, two sequences deeply branching within the Euryarchaeota were 240 retrieved. Both sequences were assembled from dataset mgm4537093.3, originating from a marine 241 sediment sample at the oil seeps of the coast of Santa Barbara, California. One of these sequences is basal 242 to the Methanomassiliicoccales, and distantly related to Methanonatronarchaeia, and the other sequence 243 is basal to the Methanosarcinales/Methanocellales/Methanomicrobiales cluster (Figure 2). However, the 244 organisms containing these sequences were not present in sufficient abundance in their respective 
245 samples to extract a draft genome from the metagenome, leaving the taxonomic association of these

246 divergent sequences unclear. Furthermore, several sequences associated with anaerobic methanotrophic

247 (ANME) archaea were retrieved (Figure 2), including a sequence from a South African gold mine (Lau et

248 al., 2014) related to ANME-1, and the first full length ANME-3 mcrA sequence from a dataset obtained

249 from the deep-sea Haakon Mosby mud volcano, the site where this group was originally discovered

250 (Niemann et al., 2006; Lösekann et al., 2007). Our analysis also substantially expands the known diversity

251 of mcrA sequences of methanogenic clades within the Euryarchaeota (Figure 2) and allows for an estimate

252 of environmental abundance of these clades. Such an abundance estimate is likely affected by sampling

253 bias, for example by an overrepresentation of biogas fermentation reactors. There are clear differences

254 comparing an abundance estimate based on PCR products in the NR (Supplemental Figure 1) with an

255 estimate by metagenomic reads (Figure 2) possibly due to primer bias, or a consequence of dereplication

256 of the PCR products in the NR, either by NCBI (removing redundant sequences) or prior to submission.

257 Aligning the BSR-filtered reads from our analysis to the mcrA database amended with the newly

258 assembled sequences shows higher average sequence identity of the aligned sequences after addition of

259 the new sequences. Before our analysis, 555,598 reads ( $29.5 \%$ of BSR-filtered reads) had lower than $90 \%$

260 identity to a sequence in our reference database, whereas after addition of the newly retrieved sequences

261 that number dropped to 313,904 reads below $90 \%$ identity (16.6\% of BSR-filtered reads; Figure 2;

262 Supplemental Figure 3). For the PCR amplicons present in the NCBI-nr (Supplemental Figure 1) these

263 numbers were comparable, with 4628 (29.1\%) sequences below 90\% identity to any sequence in the

264 database before including the newly retrieved sequences, and 2841 (17.9\%) below 90\% identity

265 afterwards. Although this is an improvement, the high number of reads still unassigned (>90\% sequence

266 identity to any database sequence) does indicate there are yet more divergent mcrA variants to be

267 discovered (Supplemental Figure 3). This highlights the potential for ongoing exploration of metagenomic

268 sequencing data as it becomes available, using our sequence-identity based analysis, and an HMM based 
approach (Boyd et al., 2018). The highest number of novel sequences retrieved in our survey of the SRA and MG-RAST was associated with the Methanomassiliicoccales, bringing the known diversity within this recently discovered order on par with that of more intensively studied groups.

272

273 Environmental distribution of the Methanomassiliicoccales

274 To further investigate the diversity and environmental distribution of the Methanomassiliicoccales, we retrieved all 116 mcrA sequences over 300 amino acids belonging to this clade from our analysis and assessed their environmental origin as documented in the SRA or MG-RAST metadata record. This analysis confirmed the presence of three major clades ('GIT', 'Environmental' and 'Lake Pavin'), but unlike the study by Söllinger et al. we do not observe clear clustering by environment (Figure 3). Even though our original dataset selection was biased against gut samples by the SRA query used, several sequences of fecal origin are represented in both the environmental and GIT clades (Figure 3). Conversely, both the 'Environmental' and 'GIT' clades contain many sequences originating from the same environment; a single study that characterizes the microbial diversity in open fermentation pits for liquor production (Guo et al., 2014). None of the assembled sequences originated from marine sediments, implying that the Methanomassiliicoccales are not abundant in these systems, and that other archaeal clades (possibly) associated with the Thermoplasmatales are responsible for the detected BDGT lipids (Becker et al., 2016). Four sequences, of which three were nearly identical, belonging to the 'Lake Pavin' clade were retrieved in our analysis (Figure 3). As this group was previously only detected by environmental PCR, and genomic data is lacking, we focused on the three near-identical sequences for more in-depth analysis. Using the mcrA sequences as a guide, we extracted a draft genome of a representative of this clade from dataset SRR636597, originating from an oil mining tailing pond (Tan et al., 2015). This draft genome is referred to as MALP (MAssiliicoccales Lake Pavin) for the remainder of the manuscript. 
293 Genomic analysis of MALP, a representative of 'Lake Pavin' clade Methanomassiliicoccales

294 The MALP draft genome assembled and binned from dataset SRR636597 consists of 1.55 megabases on

29558 contigs, and is over $92 \%$ complete with $0 \%$ contamination, as assessed using checkM (Parks et al., 296 2015). A recent assembly and binning effort of 1550 SRA datasets recovered a highly similar MAG, likely 297 representing the same microbial population, designated UBA248 (Parks et al., 2017). At an estimated 89\% 298 completeness, $1.6 \%$ contamination, and on 138 contigs UBA248 is somewhat more fragmented and 299 slightly lower quality. Furthermore, Parks et al. did not perform any analysis on this specific MAG. In 300 addition, Parks et al. also assembled a related MAG, designated UBA472, from a different site. Contig 301 alignment using Mauve (Rissman et al., 2009) and comparison of gene content using anvi'o (Eren et al., 302 2015) supports that MALP and UBA248 represent the same population, whereas UBA472 likely represents 303 a closely related organism (Supplemental Figure 4).

304 The size of the MALP genome falls within the range of previously obtained Methanomassiliicoccales 305 genomes (1.4-2.6 Mbp; Borrel et al., 2014; Lang et al., 2015). As in the other Methanomassiliicoccales, the 306 ribosomal rRNA genes in MALP are not organized in an operon, and it encodes two copies of the 5S rRNA 307 gene. Our mcrA analysis and BLAST searches using the MALP 16S rRNA gene indicate that organisms 308 closely related to MALP (>98\% 16S rRNA gene identity) are previously found in (contaminated) sediments 309 and wastewater treatment systems, but not in fecal samples.

310 MALP encodes all genes required for hydrogen dependent reduction of methanol to methane as proposed

311 in the other Methanomassiliicoccales (Figure 4A). These include the methanol:CoM methyltransferases

$312(m \operatorname{ta} A B C)$ for formation of methyl-CoM (Sauer and Thauer, 1999), methyl-CoM reductase (mcrABG) to 313 release methane and form the CoM-CoB heterodisulfide (Ermler et al., 1997), soluble heterodisulfide

314 reductase $(h d r A B C)$ and [NiFe]-hydrogenase $(m v h A G D)$ to reduce ferredoxin and heterodisulfide coupled 315 to hydrogen oxidation (Thauer et al., 2008; Wagner et al., 2017), and the Fpo-like complex 316 (fpoABCDHIJKLMN) proposed to oxidize ferredoxin and establish a proton gradient (Welte and 
317 Deppenmeier, 2011), potentially coupled to heterodisulfide reduction using hdrD (Figure 4) (Lang et al.,

318 2015). Like "Candidatus Methanomethylophilus alvus", MALP does not encode an energy conserving

319 hydrogenase to couple ferredoxin oxidation to hydrogen formation and the buildup of a proton gradient

320 (Borrel et al., 2014).

321 From both physiological studies on the Methanomassiliicoccus luminyensis culture and the "Candidatus

322 Methanomethylophilus alvus" enrichment, as well as comparative genomics, it has become clear that

323 previously enriched Methanomassiliicoccales are also capable of growth on methylamines (Borrel et al.,

324 2014; Lang et al., 2015). Metabolizing methylamines requires pyrrolysine-containing methyl transferases

$325(\mathrm{mtmBC} / \mathrm{mtbBC} / \mathrm{mttBC})$, which are present in the genomes of the previously sequenced

326 Methanomassiliicoccales. In contrast, the reconstructed MALP genome does not encode the pyrrolysine-

327 containing methyltransferases, the operon for pyrrolysine biosynthesis, the pyrrolysine tRNA synthetase,

328 or the pyrrolysine tRNA. Considering that MALP is a representative of the most basal cluster of

329 Methanomassiliicoccales (Figure 3), the absence of pyrrolysine usage from the genome suggests the

330 ability to generate methane from methylated amines was acquired recently within the

331 Methanomassiliicoccales order. In agreement with this, a previous comparative genomics study found

332 that the number of pyrrolysine containing genes in other sequenced Methanomassiliicoccales varied

333 between 3 (Methanomassiliicoccus luminyensis) and 19 ('Candidatus Methanomethylophilus alvus')

334 (Borrel et al., 2014).

335 Another unexpected feature of the MALP genome was the presence of the two catalytic subunits of N5-

336 methyltetrahydromethanopterin:CoM methyltransferase (mtrAH) (Wagner et al., 2016). In $\mathrm{CO}_{2}$-reducing

337 methanogens methyltetrahydromethanopterin:CoM methyltransferase is an eight-subunit membrane-

338 associated complex that catalyzes the transfer of a methyl group from tetrahydromethanopterin (THMPT)

339 to coenzyme $\mathrm{M}$, coupled to translocation of a sodium ion. However, only the catalytic subunits $(m t r A H)$,

340 and none of the membrane associated subunits (mtrB-G), are present in the MALP genome. This is 
341 surprising, as there is no known role for THMPT in Methanomassiliicoccales, including MALP. In contrast,

342 MALP does encode the complete C1-tetrahydrofolate (THF) pathway. The mtrA subunit, which donates

343 the methyl group to Coenzyme $\mathrm{M}$ is conserved, but contains an unusual C-terminal extension. On the

344 other hand, the $m$ trH subunit that is responsible for the transfer of the methyl group from THMPT is

345 divergent from the $\mathrm{mtrH}$ of $\mathrm{CO}_{2}$-reducing methanogens. Considering the likely absence of THMPT from

346 MALP, and the divergent $m t r H$ subunit, we propose this $m t r A H$ may be a N5-methyltetrahydrofolate:CoM

347 methyltransferase instead (Figure 4B).

348 In addition to the mtrAH genes, MALP encodes acetyl-CoA synthetase for the formation of acetyl-CoA

349 from acetate (Jetten et al., 1989), and acetyl-CoA synthase/CO dehydrogenase for the disproportionation

350 of acetyl-CoA into methyl-tetrahydromethanopterin or methyl-tetrahydrofolate and $\mathrm{CO}$, and ferredoxin

351 dependent oxidation of $\mathrm{CO}$ to $\mathrm{CO}_{2}$ (Figure 4B) (Ferry, 1992). This gene set, combined with the presence of

352 the Fpo-like complex, in theory provides MALP with a complete pathway for aceticlastic methanogenesis,

353 similar to the pathway observed in Methanosaeta thermophila (Figure 4B) (Welte and Deppenmeier,

354 2014). Even though genes required for aceticlastic methanogenesis were detected, there are a number of

355 differences between the pathway in the obligate aceticlastic methanogen Methanosaeta thermophila,

356 and the hypothetical pathway in MALP that make it doubtful that MALP has the capability to produce

357 methane from acetate.

358 Notably, MALP lacks the membrane complexes thought to conserve energy for ATP production in

359 Methanosaeta thermophila (Welte and Deppenmeier, 2014); the membrane subunits of the mtr complex

360 (mtrBCDEFG) and the membrane-bound heterodisulfide reductase ( $H d r D E)$ are absent. More specifically,

361 the 2 ATP equivalents expended in the conversion of acetate to acetyl-CoA using acetyl-CoA synthetase

362 require a minimum translocation of 7 protons/sodium atoms to regenerate the ATP (at 3 charge

363 translocations/ATP) and build up a potential of 1 net proton/sodium atom per molecule of acetate. $M$.

364 thermophila might achieve this by translocating $2 \mathrm{Na}^{+}$atoms using the $m t r$ complex, 2 protons using the 
365 membrane-bound heterodisulfide reductase $(H d r D E)$, and 3 protons using the Fpo-like complex (Welte

366 and Deppenmeier, 2014). Compared to M. thermophila, MALP lacks the sodium translocating subunits of

$367 m \operatorname{tr}(m \operatorname{tr} B C D E F G)$ and the integral membrane subunit of the membrane-bound heterodisulfide reductase

368 (HdrE). However, MALP does encode an energy conserving pyrophosphatase, and the hdrD subunit of

369 membrane bound heterodisulfide reductase has been proposed to interact with the Fpo-like complex,

370 potentially raising the number of protons translocated by the Fpo complex to four (Lang et al., 2015). In

371 addition, MALP encodes an "energy-conserving hydrogenase related" (ehr) complex, first observed in

372 Geobacter sulfurreducens (Coppi, 2005). The function of this complex is unknown, but it harbors several

373 proton-pumping subunits (Marreiros et al., 2013) and could be involved in energy conservation in MALP.

374 Although these complexes could account for sufficient charge translocation, there is not enough

375 biochemical evidence to confidently predict MALP has the ability to produce methane from acetate.

376 In addition to a pathway for aceticlastic methanogenesis, the presence of the $m t r A H$ genes, combined

377 with the pathway for N5-methyltetrahydrofolate oxidation to formate, could enable MALP to grow using

378 an unconventional type of methanol disproportionation shown in equation 1 and Figure 4C.

379

(1) $\quad 3 \mathrm{CH}_{3} \mathrm{OH}-->\mathrm{HCOO}^{-}+\mathrm{H}^{+}+2 \mathrm{CH}_{4}+\mathrm{H}_{2} \mathrm{O} \quad\left(\Delta \mathrm{G}^{\prime}=-203.4 \mathrm{~kJ} / \mathrm{mol}\right)$

380

(2) $\quad 4 \mathrm{CH}_{3} \mathrm{OH}-->\mathrm{CO}_{2}+3 \mathrm{CH}_{4}+2 \mathrm{H}_{2} \mathrm{O}$

$\left(\Delta \mathrm{G}^{0^{\prime}}=-319.4 \mathrm{~kJ} / \mathrm{mol}\right)$

381 Although thermodynamically feasible (based on $\Delta \mathrm{G}_{\mathrm{f}}{ }^{0}$ from Thauer et al., 1977), there are various caveats

382 to this proposed metabolism. First, it is less energetically favorable than methanol disproportionation to

383 methane and $\mathrm{CO}_{2}$ (equation 2), suggesting that the metabolism would not be competitive in the

384 environment. However, when expressed per mol substrate (methanol) the energy difference drops to

$38567.8 \mathrm{~kJ} / \mathrm{mol}$ methanol versus $79.85 \mathrm{~kJ} / \mathrm{mol}$ methanol for disproportionation to formate/methane and

$386 \mathrm{CO}_{2}$ /methane respectively. This relatively small difference could be overcome by efficient formate

387 removal by other organisms in the environment. 
388 Another caveat is that the endergonic methyl transfer from Coenzyme M to THMPT in methanol

389 disproportionating methanogens is thought to be driven by dissipation of a sodium gradient. As $m t r A H$ in

390 MALP is likely not membrane associated, this mechanism seems unlikely. However, methyl transfer from

391 CoM to tetrahydrofolate (THF) is likely less endergonic than transfer to THMPT (Chistoserdova et al., 1998;

392 Maden, 2000) and might proceed without being driven by a sodium gradient. The remainder of the C1-

393 THF pathway is reversible, albeit less favorable than the C1-THMPT pathway (Maden, 2000). The entire

394 C1-THF pathway is also present in Methanomassiliicoccus lumiyensis and Methanomassiliicoccus

395 intestinalis, while the other Methanomassiliicoccales lack only the gene for conversion between N5-

396 methyltetrahydrofolate and 5-10 methylenetetrahydrofolate (metF). This pathway is also proposed to be

397 used in the oxidative direction, to supply intermediates for purine biosynthesis (Lang et al., 2015). None

398 of the Methanomassiliicoccales, including MALP, encode formate dehydrogenase (Lang et al., 2015).

399 Oxidation of the methyl group would thus stop at formate, generating 4 electrons and resulting in the

400 stoichiometry shown in equation (1). A final caveat with this proposed pathway is the conversion between

$401 \mathrm{NAD}(\mathrm{P}) \mathrm{H}$ generated in the oxidation of N5-methyltetrahydrofolate to formate, and the ferredoxin that is

402 oxidized at the Fpo-like complex. MALP does not encode a homolog of Ferredoxin:NADP reductase, thus

403 at present the candidate for this reaction is unknown.

404 In summary, the MALP genome, belonging to the 'Lake Pavin' clade of the Methanomassiliicoccales,

405 indicates that the MALP organism is a hydrogenotrophic methyl-reducing methanogen, capable of growth

406 on methanol. Unlike the other members of the Methanomassiliicoccales, MALP does not encode the

407 genes required for growth on other methylated compounds, such as methylamines or methylsulfides.

408 However, MALP does encode an unusual mtrAH complex, which might allow for aceticlastic

409 methanogenesis, as well as methanol disproportionation. However, as outlined above, the latter two

410 predicted metabolic capabilities are highly uncertain without further physiological and biochemical data. 
411 Therefore, obtaining a cultured representative of this clade would greatly aid in testing these 412 hypotheses. 


\section{Conclusions}

414

415 Metagenomic marker gene mining is a complementary approach to genome resolved metagenomics, and

416 can be used to assess phylogenetic diversity and environmental distribution of a microbial process. Due

417 to the size (and continued rapid growth) of public sequence databases our implementation is slow.

418 However, this approach is broadly applicable as it requires minimal computational power due to the small

419 database size, and minimal storage (in contrast to locally storing a version of the public databases)

420 because datasets are processed sequentially and then deleted. Using this marker gene mining approach,

421 we recovered novel mcrA gene diversity, and identified promising targets for more in-depth analysis

422 leading to better understanding of the habitat distribution and metabolic versatility of the environmental

423 Methanomassiliicoccales. Marker gene mining can be used to query the large amount of data from the

424 many publicly available sequencing projects for specific questions, and potentially lead to discoveries

425 outside the scope of the original studies. The rapidly increased throughput, and reduced cost, of next

426 generation sequencing ensures that much more data will become available in years to come, and

427 complementary strategies to analyze this sequencing data effectively will be increasingly important going 428 forward.

\section{Acknowledgements}

432 We thank Woody Fischer and Connor Skennerton for helpful discussion and Grayson Chadwick for 433 critically reading the manuscript. 
436

437

438

439

440

441

442

443

444

445

446

447

448

449

450

451

452

453

454

455

456

457

458

459

460

461

462

463

464

465

466

63

\section{References}

Albertsen M, Hugenholtz P, Skarshewski A, Nielsen KL, Tyson GW, Nielsen PH, (2013). Genome sequences of rare, uncultured bacteria obtained by differential coverage binning of multiple metagenomes. Nature biotechnology, 31(6), p.533.

Bankevich A, Nurk S, Antipov D, Gurevich AA, Dvorkin M, Kulikov AS, Lesin VM, Nikolenko SI, Pham S, Prjibelski AD, Pyshkin AV. (2012). SPAdes: a new genome assembly algorithm and its applications to singlecell sequencing. Journal of Computational Biology 19: 455-477.

Becker KW, Elling FJ, Yoshinaga MY, Söllinger A, Urich T, Hinrichs K-U. (2016). Unusual Butane- and Pentanetriol-Based Tetraether Lipids in Methanomassiliicoccus luminyensis, a Representative of the Seventh Order of Methanogens Appl Environ Microbiol 82: 4505-4516.

Borrel G, Harris HMB, Parisot N, Gaci N, Tottey W, Mihajlovski A, Deane J, Gribaldo S, Bardot O, Peyretaillade E, Peyret P. (2013a). Genome Sequence of 'Candidatus Methanomassiliicoccus intestinalis' Issoire-Mx1, a Third Thermoplasmatales-Related Methanogenic Archaeon from Human Feces. Genome Announc 1 doi:10.1128/genomeA.00453-13.

Borrel G, Harris HMB, Tottey W, Mihajlovski A, Parisot N, Peyretaillade E, Peyret P, Gribaldo S, O'Toole PW, Brugère JF. (2012). Genome Sequence of 'Candidatus Methanomethylophilus alvus' Mx1201, a Methanogenic Archaeon from the Human Gut Belonging to a Seventh Order of Methanogens. Journal of bacteriology 194: 6944-6945.

Borrel G, O'Toole PW, Harris HMB, Peyret P, Brugère J-F, Gribaldo S. (2013b). Phylogenomic data support a seventh order of Methylotrophic methanogens and provide insights into the evolution of Methanogenesis. Genome Biol Evol 5: 1769-1780.

Borrel G, Parisot N, Harris HMB, Peyretaillade E, Gaci N, Tottey W, Bardot O, Raymann K, Gribaldo S, Peyret P, O'Toole PW. (2014). Comparative genomics highlights the unique biology of Methanomassiliicoccales, a Thermoplasmatales-related seventh order of methanogenic archaea that encodes pyrrolysine $B M C$ Genomics 15: 679 
468 Boyd, J. A., Woodcroft, B. J., \& Tyson, G. W. (2018). GraftM: a tool for scalable, phylogenetically

Buchfink B, Xie C, Huson DH. (2014). Fast and sensitive protein alignment using DIAMOND. Nature

Carver T, Harris SR, Berriman M, Parkhill J, McQuillan JA. (2012). Artemis: an integrated platform for visualization and analysis of high-throughput sequence-based experimental data. Bioinformatics 28: 464469.

Chistoserdova L, Vorholt JA, Thauer RK, Lidstrom ME. (1998) C1 transfer enzymes and coenzymes linking methylotrophic bacteria and methanogenic Archaea. Science 281: 99-102

Coppi MV. (2005). The hydrogenases of Geobacter sulfurreducens: a comparative genomic perspective. Microbiology 151: 1239-1254.

Daims H, Lebedeva EV, Pjevac P, Han P, Herbold C, Albertsen M, Jehmlich N, Palatinszky M, Vierheilig J, nitrification by Nitrospira bacteria. Nature 528: 504-509. populations of Planctomycetes and Proteobacteria are abundant in the surface ocean. bioRxiv. doi:https://doi.org/10.1101/129791

492

Denonfoux J, Parisot N, Dugat-Bony E, Biderre-Petit C, Boucher D, Morgavi DP, Le Paslier D, Peyretaillade

494 E, Peyret P. (2013). Gene capture coupled to high-throughput sequencing as a strategy for targeted 495 metagenome exploration. DNA Res 20: 185-196.

496

497 Dick GJ, Andersson AF, Baker BJ, Simmons SL, Thomas BC, Yelton AP, Banfield JF (2009). Community498 wide analysis of microbial genome sequence signatures. Genome biology, 10(8) 
499

500 Dridi B, Fardeau ML, Ollivier B, Raoult D, Drancourt M. (2012). Methanomassiliicoccus luminyensis gen.

501 nov., sp. nov., a methanogenic archaeon isolated from human faeces. Int J Syst Evol Microbiol 62: 1902-

5021907.

503

504 Edgar RC. (2004). MUSCLE: multiple sequence alignment with high accuracy and high throughput. Nucleic

505 Acids Res 32: 1792-1797

506

507 Edgar RC. (2010). Search and clustering orders of magnitude faster than BLAST. Bioinformatics 26: 24605082461.

509

510 Eren AM, Esen ÖC, Quince C, Vineis JH, Morrison HG, Sogin ML, Delmont TO (2015) Anvi'o: an advanced 511 analysis and visualization platform for 'omics data. PeerJ 3: e1319.

512

513 Ermler U, Grabarse W, Shima S, Goubeaud M, Thauer RK. (1997). Crystal structure of methyl-coenzyme M 514 reductase: the key enzyme of biological methane formation. Science 278: 1457-1462.

515

516 Evans PN, Parks DH, Chadwick GL, Robbins SJ, Orphan VJ, Golding SD, Tyson GW (2015). Methane

517 metabolism in the archaeal phylum Bathyarchaeota revealed by genome-centric metagenomics. Science 518 350: 434-438.

519

520

Ferry JG. (1992). Methane from acetate. Journal of bacteriology 174: 5489-5495.

521

522 Finn RD, Bateman A, Clements J, Coggill P, Eberhardt RY, Eddy SR, Heger A, Hetherington K, Holm L, Mistry

523 J, Sonnhammer EL. (2013). Pfam: the protein families database. Nucleic Acids Res 42: D222-D230.

524

525 Großkopf R, Stubner S, Liesack W. (1998). Novel Euryarchaeotal lineages detected on rice roots and in the 526 anoxic bulk soil of flooded rice microcosms. Appl Env Microbiol 64: 4983-4989.

527

528 Guo M-Y, Huo D-Q, Ghai R, Rodriguez-Valera F, Shen C-H, Zhang N, Zhang SY, Hou CJ. (2014).

529 Metagenomics of Ancient Fermentation Pits Used for the Production of Chinese Strong-Aroma Liquor.

530 Genome announcements 2.5 (2014): e01045-14 
532 Hug LA, Baker BJ, Anantharaman K, Brown CT, Probst AJ, Castelle CJ, Butterfield CN, Hernsdorf AW, Amano

533 Y, Ise K, Suzuki Y, Dudek N, Relman DA, Finstad KM, Amundson R, Thomas BC, Banfield JF. (2016). A new

534 view of the tree of life Nature microbiology 1: 16048.

535

536 Hyatt D, Chen G-L, LoCascio PF, Land ML, Larimer FW, Hauser L. (2010). Prodigal: prokaryotic gene 537 recognition and translation initiation site identification. BMC Bioinformatics 11: 119.

538

lino T, Tamaki H, Tamazawa S, Ueno Y, Ohkuma M, Suzuki KI, Igarashi Y, Haruta S. (2013) 'Candidatus Methanogranum caenicola': a novel methanogen from the anaerobic digested sludge, and proposal of Methanomassiliicoccaceae fam. nov. and Methanomassiliicoccales ord. nov., for a methanogenic lineage of the class Thermoplasmata. Microbes and environments 28: 244-50.

543

Jetten MS, Stams AJ, Zehnder AJ. (1989). Isolation and characterization of acetyl-coenzyme A synthetase from Methanothrix soehngenii. Journal of bacteriology 171: 5430-5435.

Knittel K, Boetius A. (2009). Anaerobic Oxidation of Methane: Progress with an Unknown Process. Annu Rev Microbiol 63: 311-334.

Kodama Y, Shumway M, Leinonen R, International Nucleotide Sequence Database Collaboration. (2012). The Sequence Read Archive: explosive growth of sequencing data. Nucleic Acids Res 40: D54-6.

Lang K, Schuldes J, Klingl A, Poehlein A, Daniel R, Brune A. (2015). New mode of energy metabolism in the seventh order of methanogens as revealed by comparative genome analysis of 'Candidatus methanoplasma termitum'. Appl Environ Microbiol 81: 1338-1352. HE, Riedel D, Richnow HH, Adrian L, Reemtsma T, Lechtenfeld OJ, Musat F. (2016). Thermophilic archaea activate butane via alkyl-coenzyme M formation. Nature 539: 396-401.

560

561 Lau MCY, Cameron C, Magnabosco C, Brown CT, Schilkey F, Grim S, Hendrickson S, Pullin M, Sherwood

562 Lollar B, van Heerden E, Kieft TL. (2014). Phylogeny and phylogeography of functional genes shared among 
563 seven terrestrial subsurface metagenomes reveal $\mathrm{N}$-cycling and microbial evolutionary relationships.

564 Frontiers in Microbiology 5: 531.

565

566 Le SQ, Dang CC, Gascuel O. (2012) Modeling protein evolution with several amino acid replacement

567 matrices depending on site rates. Molecular biology and evolution, 29(10), 2921-2936.

568

569

Lechner M, Findeiß S, Steiner L, Marz M, Stadler PF, Prohaska SJ. (2011). Proteinortho: detection of (co-

570

Jorthologs in large-scale analysis. BMC Bioinformatics 12: 124.

571

572

Letunic I, Bork P. (2016). Interactive tree of life (iTOL) v3: an online tool for the display and annotation of

573 phylogenetic and other trees. Nucleic Acids Res 44: W242-5.

574

575 Li D, Liu C-M, Luo R, Sadakane K, Lam T-W. (2015). MEGAHIT: An ultra-fast single-node solution for large 576 and complex metagenomics assembly via succinct de Bruijn graph. Bioinformatics. 31: 1674-1676.

577

578

Lösekann T, Knittel K, Nadalig T, Fuchs B, Niemann H, Boetius A, Amann R (2007). Diversity and abundance of aerobic and anaerobic methane oxidizers at the Haakon Mosby Mud Volcano, Barents Sea. Appl Environ Microbiol 73: 3348-3362.

581

582

Lüke C, Speth DR, Kox MAR, Villanueva L, Jetten MSM. (2016). Metagenomic analysis of nitrogen and methane cycling in the Arabian Sea oxygen minimum zone. PeerJ 4: e1924.

584

Maden BE. (2000). Tetrahydrofolate and tetrahydromethanopterin compared: functionally distinct carriers in C1 metabolism. Biochem J 350 Pt 3: 609-629.

587

588

Marreiros BC, Batista AP, Duarte AMS, Pereira MM. (2013). A missing link between complex I and group 4 membrane-bound [NiFe]-hydrogenases. Biochim Biophys Acta 1827: 198-209.

590

591

Meyer F, Paarmann D, D'souza M, Olson R, Glass EM, Kubal M, Paczian T, Rodriguez A, Stevens R, Wilke A, Wilkening J. (2008). The metagenomics RAST server - a public resource for the automatic phylogenetic and functional analysis of metagenomes. BMC Bioinformatics 9: 386. 
595 Mondav R, Woodcroft BJ, Kim E-H, McCalley CK, Hodgkins SB, Crill PM, Chanton J, Hurst GB, VerBerkmoes

596 NC, Saleska SR, Hugenholtz P, Rich VI, Tyson GW. (2014). Discovery of a novel methanogen prevalent in

597 thawing permafrost. Nat Comms 5: 3212.

598

599 Nagle DP, Wolfe RS, 1983. Component A of the methyl coenzyme M methylreductase system of

600 Methanobacterium: resolution into four components. Proc. Natl. Acad. Sci. 80(8), 2151-2155.

601

602 Niemann H, Lösekann T, de Beer D, Elvert M, Nadalig T, Knittel K, Amann R, Sauter EJ, Schlüter M, Klages

$603 \mathrm{M}$, Foucher JP, Boetius A. (2006). Novel microbial communities of the Haakon Mosby mud volcano and 604 their role as a methane sink. Nature 443: 854-858.

605

606

Parks D, Rinke C, Chuvochina M, Chaumeil P-A, Woodcroft BJ, Evans PN, Hugenholtz P, Tyson GW. (2017).

607

Recovery of nearly 8,000 metagenome-assembled genomes substantially expands the tree of life. Nat

608

Microbiol 2: 1533-1542.

609

610 Parks DH, Imelfort M, Skennerton CT, Hugenholtz P, Tyson GW. (2015). CheckM: assessing the quality of

611 microbial genomes recovered from isolates, single cells, and metagenomes. Genome Research 25: 1043-

6121055.

613

614 Paul K, Nonoh JO, Mikulski L, Brune A. (2012). 'Methanoplasmatales,' Thermoplasmatales-related archaea 615 in termite guts and other environments, are the seventh order of methanogens. Appl Env Microbiol 78: 616 8245-53.

617

618 Peng Y, Leung HCM, Yiu SM, Chin FYL. (2012). IDBA-UD: a de novo assembler for single-cell and 619 metagenomic sequencing data with highly uneven depth. Bioinformatics 28: 1420-1428.

620

621 Rasko DA, Myers GS, Ravel J. (2005). Visualization of comparative genomic analyses by BLAST score ratio.

622 BMC Bioinformatics 6: 2.

623

624 Rissman AI, Mau B, Biehl BS, Darling AE, Glasner JD, Perna NT. (2009). Reordering contigs of draft genomes 625 using the Mauve Aligner. Bioinformatics 25: 2071-2073.

626 
627 Sauer K, Thauer RK. (1999). Methanol:coenzyme-M methyltransferase from Methanosarcina barkeri -628 substitution of the corrinoid harbouring subunit MtaC by free cob(I)alamin. European Journal of 629 Biochemistry 261: 674-681.

630

631 Scheller S, Goenrich M, Boecher R, Thauer RK, Jaun B. (2010). The key nickel enzyme of methanogenesis 632 catalyses the anaerobic oxidation of methane. Nature 465: 606-608.

633

Seemann T. (2014). Prokka: rapid prokaryotic genome annotation. Bioinformatics 30: 2068-2069.

635

Sorokin DY, Makarova KS, Abbas B, Ferrer M, Golyshin PN, Galinski EA, Ciordia S, Mena MC, Merkel AY, 637 Wolf YI, van Loosdrecht MCM, Koonin EV. (2017). Discovery of extremely halophilic, methyl-reducing euryarchaea provides insights into the evolutionary origin of methanogenesis. Nat Microbiol 2: 17081.

639

Söllinger A, Schwab C, Weinmaier T, Loy A, Tveit AT, Schleper C, Urich T. (2016). Phylogenetic and genomic analysis of Methanomassiliicoccales in wetlands and animal intestinal tracts reveals clade-specific habitat preferences. FEMS Microbiology Ecology 92: fiv149.

643

Speth DR, In 't Zandt MH, Guerrero-Cruz S, Dutilh BE, Jetten MSM. (2016). Genome-based microbial 645 ecology of anammox granules in a full-scale wastewater treatment system. Nat Comms 7: 11172.

646

Stamatakis A. (2014). RAxML version 8: a tool for phylogenetic analysis and post-analysis of large phylogenies. Bioinformatics 30: 1312-1313.

649

Tajima K, Nagamine T, Matsui H, Nakamura M, Aminov RI. (2001). Phylogenetic analysis of archaeal $16 \mathrm{~S}$ rRNA libraries from the rumen suggests the existence of a novel group of archaea not associated with known methanogens. FEMS Microbiology Letters 200: 67-72.

653

654 Tan B, Fowler SJ, Abu Laban N, Dong X, Sensen CW, Foght J, Gieg LM. (2015). Comparative analysis of 655 metagenomes from three methanogenic hydrocarbon-degrading enrichment cultures with 41 656 environmental samples. The ISME Journal 9: 2028-2045. 
658 Thauer RK, Jungermann K, Decker K. (1977). Energy conservation in chemotrophic anaerobic bacteria.

659 Bacteriol Rev 41: 809-180.

660

661 Thauer RK, Kaster A-K, Seedorf H, Buckel W, Hedderich R. (2008). Methanogenic archaea: ecologically 662 relevant differences in energy conservation. Nat Rev Micro 6: 579-591.

663

664 Thomas T, Gilbert J, Meyer F. (2012). Metagenomics - a guide from sampling to data analysis. Microb 665 Inform Exp 2: 3.

666

667 Tyson GW, Chapman J, Hugenholtz P, Allen EE, Ram RJ, Richardson PM, Solovyev VV, Rubin EM, Rokhsar 668 DS Banfield JF. (2004). Community structure and metabolism through reconstruction of microbial

669 genomes from the environment. Nature, 428(6978)

670

671

van Kessel MAHJ, Speth DR, Albertsen M, Nielsen PH, Op den Camp HJM, Kartal B, Jetten MSM, Lücker S. 672 (2015). Complete nitrification by a single microorganism. Nature 528: 555-559.

673

674 Vanwonterghem I, Evans PN, Parks DH, Jensen PD, Woodcroft BJ, Hugenholtz P, Tyson GW. (2016). 675 Methylotrophic methanogenesis discovered in the archaeal phylum Verstraetearchaeota. Nat Microbiol 676 1: 16170.

677

678

Wagner T, Ermler U, Shima S. (2016). MtrA of the sodium ion pumping methyltransferase binds cobalamin 679 in a unique mode. Sci Rep 6: 28226.

680

681 Wagner T, Koch J, Ermler U, Shima S. (2017). Methanogenic heterodisulfide reductase (HdrABC-MvhAGD) 682 uses two noncubane [4Fe-4S] clusters for reduction. Science 357: 699-703.

683

684 Welte C, Deppenmeier U. (2014). Bioenergetics and anaerobic respiratory chains of aceticlastic 685 methanogens. Biochim Biophys Acta 1837: 1130-1147.

686

687 Welte C, Deppenmeier U. (2011). Membrane-bound electron transport in Methanosaeta thermophila. 688 Journal of bacteriology 193: 2868-2870.

689 
690 Wright A-DG, Williams AJ, Winder B, Christophersen CT, Rodgers SL, Smith KD. (2004). Molecular diversity 691 of rumen methanogens from sheep in Western Australia. Appl Env Microbiol. 70:1263-70.

692 


\section{Figure 1 (on next page)}

Overview of the marker gene mining workflow
A) Construction of the mcrA reference database using the two mcrA Pfam families and the NCBI non-redundant protein database. B) Screening metagenomes in the sequencing read archive and MG-RAST for the presence of mcrA and reconstructing mcrA gene sequences. 


\section{Figure 2 (on next page)}

Phylogeny and environmental detection of recovered mcrA sequences
A) Maximum likelihood phylogeny of the translated mcrA sequences. Background shading is used to delineate major clades. Bootstrap values over $70 \%$ are indicated by black circles. The two copies of mcrA in Methanococcales and Methanobacteriales are indicated with mcrA \& $m r t A$. B) Protein accession number or dataset accession number of the sequences in the phylogeny. Sequences obtained from the NCBI-nr are highlighted in orange, sequences assembled in this study from SRA and MG-RAST datasets are highlighted in blue. C) Number of sequences present in the NCBI-nr with over $90 \%$ amino acid identity to the sequences in the phylogeny. This includes mostly gene fragments amplified using PCR D) Number of sequencing reads, after BLAST Score Ratio (BSR) filtering, from metagenomes in the SRA and MG-RAST with over $90 \%$ amino acid identity to the sequences in the phylogeny. 


\section{A}

mcrA phylogeny

\section{B}

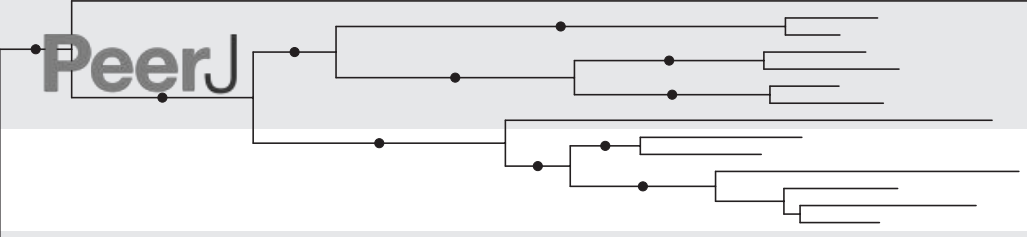

Syntrop/parchgeum USC

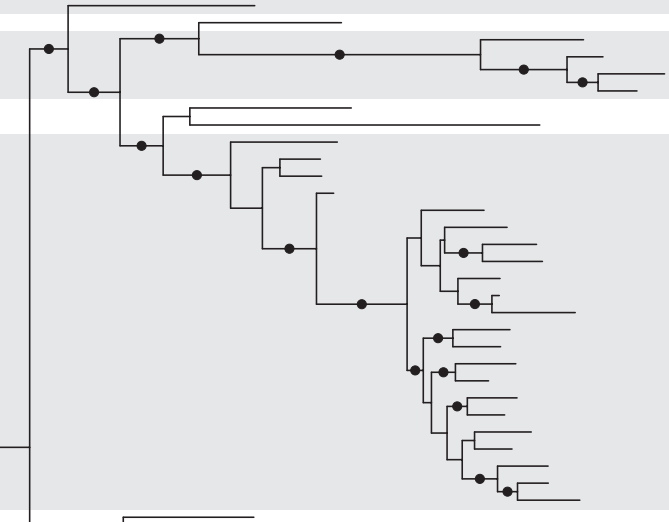

Verstraetearchaeota
Methanofastidiosales

ANME-1

Methanonatronarchaeia

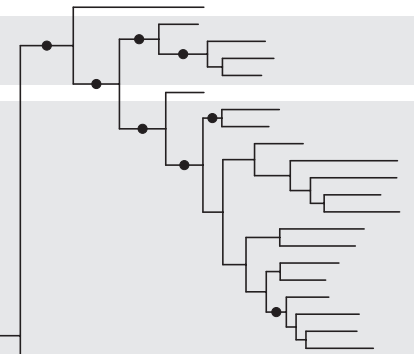

Methanopyrales

Methanococcales, mcrA

Methanococcales, mrtA

Methanobacteriales, mrtA

Methanobacteriales, mcrA

unknown lineage ANME-2a/b

Methanosaetaceae

Methermicoccaceae

ANME-2C

ANME-2d

ANME-3

Methanosarcinaceae

Methanocella

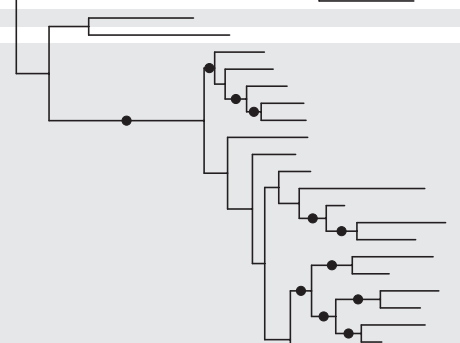

Methanoflorens

Methanomicrobiales

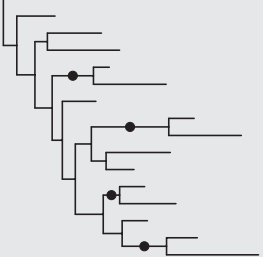

0.1

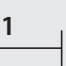

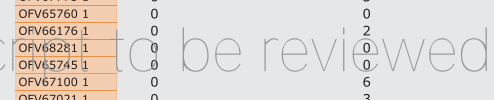

\section{年}

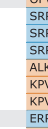

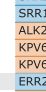

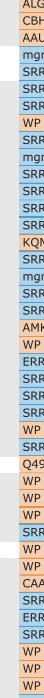

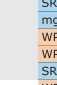

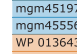

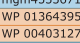

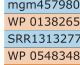

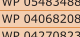

WP 012956722

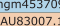

AUU83007.1
SRR2133851

ABK 143650.1
mgma491397.

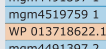

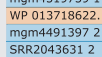

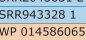
SRR 1313313181
WP 042686202.1

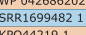

SRR3546449.1

SRR19716241

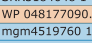

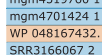

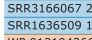

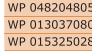

$\frac{\mathrm{K} \times \mathrm{K} \text { KS42425.1 }}{\mathrm{SRR} 13132278}$

WP 012035371

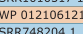

SRR13132433

AFI62041.1

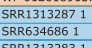

\begin{tabular}{l} 
SRRR 13132831 \\
WP D 0073314361 \\
\hline
\end{tabular}

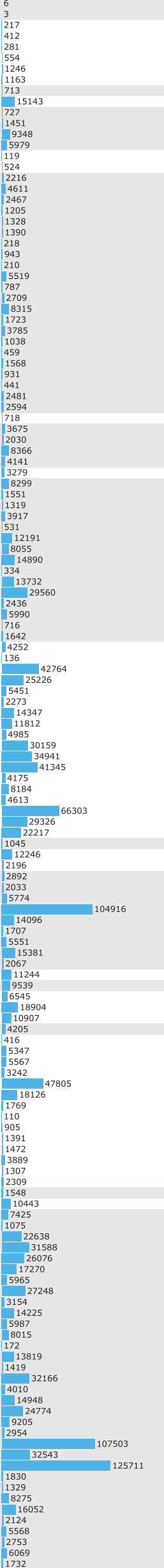




\section{Figure 3 (on next page)}

Phylogeny and environmental distribution of the mcrA sequences within the Methanomassiliicoccales order

Maximum likelihood phylogeny of the translated mcrA sequences belonging to the Methanomassiliicoccales order retrieved in this study, and the five Methanomassiliicoccales mcrA sequences present in the NCBI-nr, after dereplication at $90 \%$ identity, at the time of database construction. Bootstrap values over $70 \%$ are indicated by black circles. Leaf labels are accession numbers of the protein sequence (in the case of the five reference sequences) or source dataset. Coloring of leaf labels indicates source environment. Shading indicates the three clusters discussed in the text: (A) Lake Pavin cluster, (B) Environmental cluster, (C) Gastrointestinal tract (GIT) cluster. 


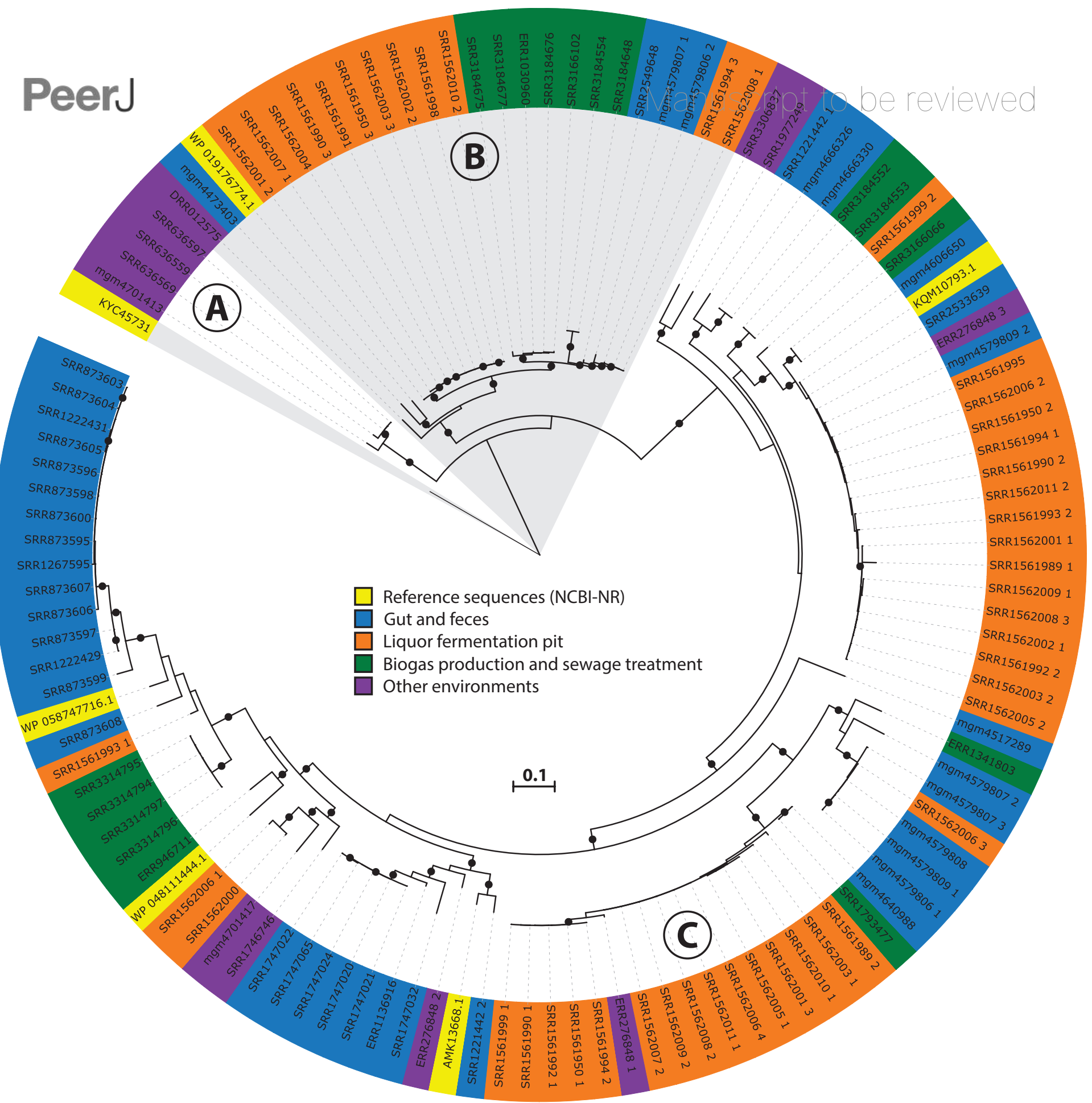




\section{Figure 4 (on next page)}

Proposed energy metabolism of the Methanomassiliicoccales Lake Pavin (MALP) cluster genome

Proposed energy metabolism in the MALP genome. A) Hydrogen dependent reduction of methanol to methane. B) Acetate disproportionation to methane and carbon dioxide C) methanol disproportionation to methane and formate. Substrates of energy metabolism are indicated in red, products in blue. Enzymes are indicated by numbered circles. 1) methanol:coM methyltransferase 2) Methyl-coenzyme M reductase 3) [NiFe]hydrogenase/heterodisulfide reductase 4) Fpo-like complex/heterodisulfide reductase 5) ATP synthase 6) Acetyl-CoA synthetase 7) Acetyl-CoA synthase 8) N5-tetrahydrofolate:Coenzyme $M$ methyltransferase 9) CO-dehydrogenase 10) Carbonic anhydrase 11) energy conserving pyrophosphatase 12) N5-methyltetrahydrofolate oxidation pathway. CoM: Coenzyme M, CoB: Coenzyme B, Fd: ferredoxin, THF: tetrahydrofolate, THMPT: tetrahydromethanopterin. 

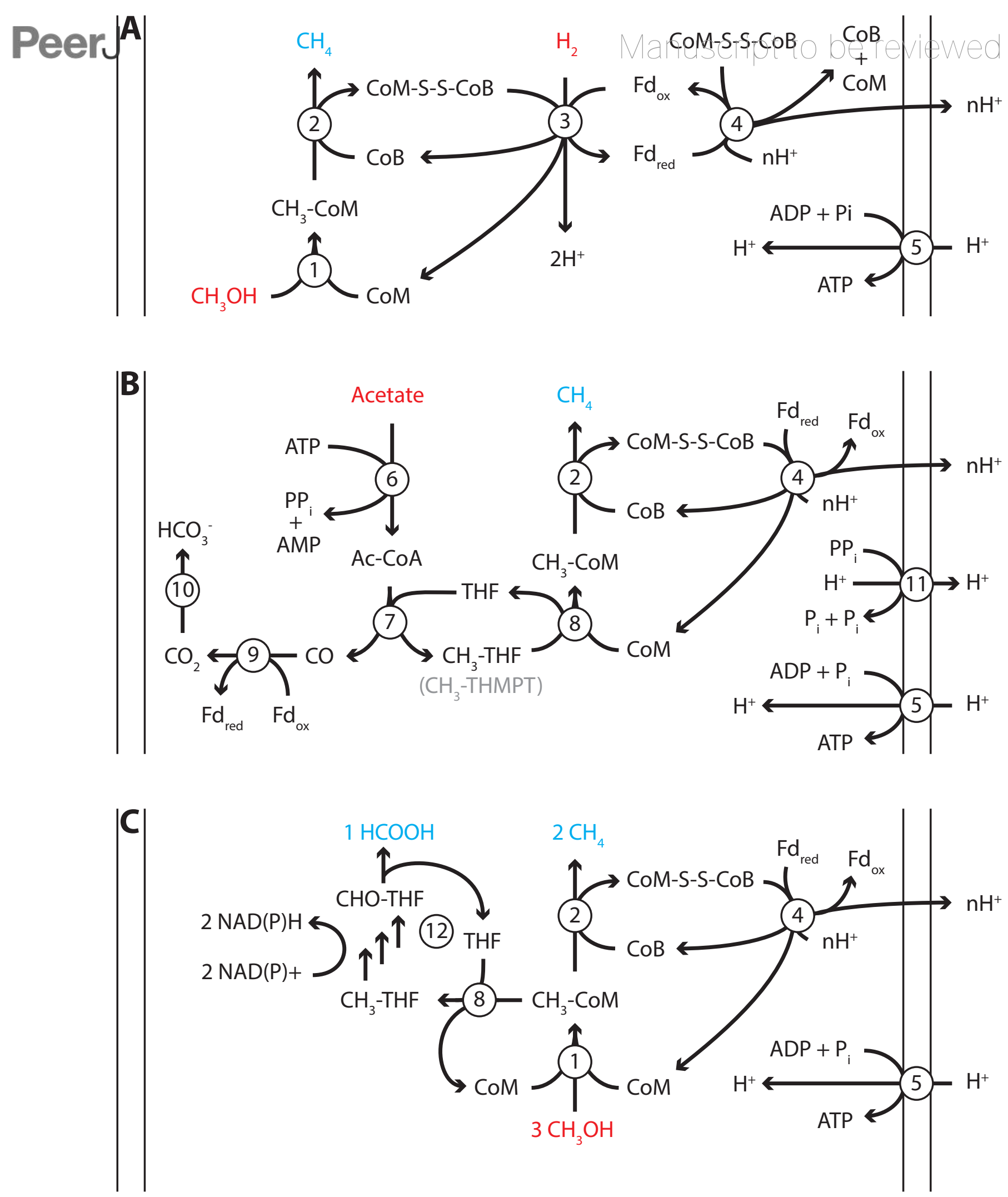